Military Technical College

Kobry El-Kobbah, Cairo, Egypt

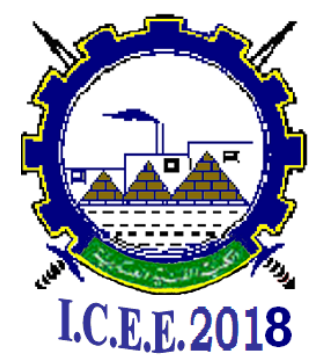

$9^{\text {th }}$ International Conference on

Chemical \& Environmental Engineering

3-5 April 2018

\title{
SMD-3
}

\section{Innovating tools for monitoring pollutants in aquatic environments}

\author{
Gaelle CATANANTE ${ }^{\text {a }}$, Akhtar HAYAT ${ }^{\text {b }}$, Jean Louis MARTY ${ }^{\text {a }}$
}

\begin{abstract}
Toxic substances such as heavy metals and environmental pollutants may contaminate the aquatic resources and portable water systems, posing serious threats to the humans, agriculture, livestock and wildlife. In the last decades, discharge of industrial effluents containing xenobiotic compounds such as pharmaceuticals, endocrine disruptors, surfactants, and industrial additives to water resources has become a point of major concern for the society. The worldwide different regulatory authorities has set the regulatory standards for these contaminants with very low permissible limits i.e. $\mathrm{pg} / \mathrm{L}$ or $\mathrm{ng} / \mathrm{kg}$. In consideration of toxicity and ubiquity of these compounds, the development of fast, sensitive and reliable detection methods is highly needed. Conventional analytical methods are commonly used to insure the aquatic environment safety. However, analytical figures of merit such as relatively higher limited of detection, lack of portability and unsuitability for on-site analysis, elevated cost and requirement of highly trained professionals limit their real time applications [1, 2], and are therefore difficult to implement specially in the developing countries. To overcome the above limitation, researchers have focused to design novel screening methods to maintain water quality.

The current tendency has driven the development of biosensors as new analytical tools with potential to provide low cost, fast, reliable and sensitive measurements, while many of them are aimed for on-site analysis. Biosensors are defined as analytical devices incorporating a biological material, or biomimic, intimately associated with or integrated within a physicochemical transducer or transducing microsystem. The main advantages of biosensors include short analysis time, low cost per assay, possible portability, suitability for real-time/ on-site measurements. Biosensors may not completely replace the official analytical methods, but can be used both by regulatory authorities and industry to add up the information for routine testing and screening of samples. These new technologies have been applied in quantitative analysis of various target analytes for diverse applications.

The present state of art suggests the evidence of several screening and biosensing methods for the detection of xeno-compounds. In this context, the current activities and results of our research group will be discussed with particular focus on the advantages and drawbacks of each developed methodologies. It is anticipated that the future will see implementation of bio sensing methods such as those based on the use of multiple array biosensors to monitor the emerging environmental contaminants.
\end{abstract}


Military Technical College

Kobry El-Kobbah, Cairo, Egypt

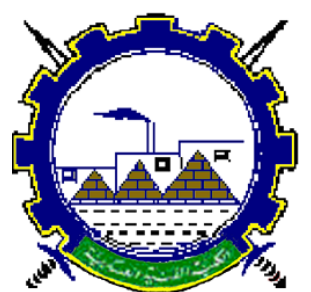

I.C.E.E.2018 $9^{\text {th }}$ International Conference

on

Chemical \& Environmental

Engineering

3-5 April 2018

1. Richardson SD, Ternes TA. Water Analysis: Emerging Contaminants and Current Issues. Analytical chemistry 2014;86:2813-48. [

2. Schymanski EL, Singer HP, Slobodnik J, Ipolyi IM, Oswald P, Krauss M, et al. Non-target screening with high-resolution mass spectrometry: critical review using a collaborative trial on water analysis. Analytical and bioanalytical chemistry 2015;407:6237-55. IIII

a - BAE-LBBM Laboratory, University of Perpignan Via Domitia, 52, Avenue Paul Alduy, 66860 Perpignan Cedex, France

b - Interdisciplinary Research Centre in Biomedical Materials (IRCBM), COMSATS Institute of Information technology, Lahore, Pakistan

Tel: +3368662254 or +33616814591 Fax:+33 468662223 E-mail : jlmarty@ univ-perp.fr 